\title{
LA REPRODUCTIBILIDAD TÉCNICA Y EL KITSCH: ECOS DE DOS DEBATES ESTÉTICOS
}

\author{
Beñat Sarasola \\ Universidad del País Vasco \\ benat.sarasola@ehu.eus
}

\begin{abstract}
RESUMEN / ABSTRACT
El presente artículo analiza de forma comparativa dos debates estéticos fundamentales del siglo XX, la reproductibilidad técnica y el kitsch. Ambos debates se desarrollaron a lo largo de las décadas 20 y 30 , y tuvieron como protagonistas, entro otros, a Adorno y Benjamin. El artículo defiende que existen numerosos elementos comunes en ambos debates, que dan buena cuenta de la controversia filosófica que tuvieron los dos filósofos. Pese a que la discusión entre ellos estalla a partir de la cuestión de la reproductibilidad técnica, en sus textos del kitsch ya se pueden observar muchos elementos que apuntan hacia esa discordancia.
\end{abstract}

Palabras Clave: reproductibilidad técnica, estética, kitsch, Adorno, Benjamin.

\section{MECHANICAL REPRODUCTION AND KITSCH: ECHOES FROM TWO AESTHETICS DEBATES}

The article analyses comparatively two essential aesthetics debates of the $X X^{\text {th }}$ century, mechanical reproduction and kitsch. Both of them were developed throughout the $20^{\text {th }}$ and $30^{\text {th }}$ decades, and had as protagonists, among others, Adorno and Benjamin. The article defends that exist many common elements between the two debates, that elucidate the philosophical controversy between the two philosophers. Despite that the discussion emerged with the mechanical reproduction issue, there are several elements in their texts about kitsch that indicate in advance the disagreement.

KEYWORDS: Mechanical Reproduction, Kitsch, Aesthetics, Adorno, Benjamin.

R⿴囗十 El debate cultural que se dio, en el periodo de entreguerras, sobre los cambios tecnológicos y sus efectos sociales fue fundamental en el desarrollo de las teorías estéticas a lo largo del siglo XX. Especialmente a partir de la década de los 70, cuando las teorías posmodernas eclosionaron y revolucionaron el panorama cultural y filosófico contemporáneo, las alusiones al debate sobre la reproductibilidad técnica y la industria cultural de los años 30 y 40 han sido constantes ${ }^{1}$. Evidentemente, los

Esta publicación forma parte de los proyectos US 17/10 (UPV-EHU) y FFI2017-84342-P (MINECO) que desarrolla el grupo de investigación IT 1047-16".

1 Dos ejemplos de ello son: Peter Bürger y su teoría de la vanguardia y Andreas Huyssen y su teoría de las dos posmodernidades. 
dos teóricos más significativos al respecto son Walter Benjamin y Theodor Adorno. El debate estético entre los dos supone una de las controversias estéticas más significativas del siglo XX (Wolin 1994, p. 163). La controversia, que parte de unas desavenencias políticas entre Adorno y Benjamin (Buck-Morss 1981, p. 302) que se evidencian ya en 1935, se alarga hasta la muerte de Benjamin en 1940. Las diferencias se manifiestan claramente en la discusión sobre la reproductibilidad técnica, y se extiende en los textos de Benjamin sobre Baudelaire. El debate de la reproductibilidad técnica, emblemático a la hora de analizar la relación entre Adorno y Benjamin, se relaciona, sin embargo, con otros debates desarrollados paralelamente o incluso anteriormente, en el que ambos teóricos participaron también de una forma u otra. Entre ellos sobresale la discusión sobre el kitsch, que es otro de los debates culturales esenciales de la primera mitad del siglo XX y que ha sido reelaborado también en el contexto de la posmodernidad. Las teorías sobre el kitsch se desarrollaron fundamentalmente en el ámbito germano, impulsadas por los textos de Hermann Broch (además de los de Adorno y Benjamin), pero llegaron también a otras latitudes de la mano de uno de los teóricos más importantes de la cuestión, el teórico de arte estadounidense Clement Greenberg.

En el presente artículo se analizarán los dos debates, el de la reproductibilidad técnica y el del kitsch, sacando a relucir las conexiones teóricas entre ambos, y enmarcándolos en las diferencias teóricas entre Adorno y Benjamin. Pese a que las ideas sobre el kitsch, tanto de uno como de otro, se desarrollan con anterioridad a la fecha del desencuentro explícito entre ambos, en ese momento ya se pueden observar unas diferencias fundamentales que posteriormente se materializarán más claramente en el debate sobre la reproductibilidad técnica.

\section{El debate sobre la reproductibilidad técnica}

El debate sobre la reproductibilidad técnica y la industria se inaugura con el célebre texto de Benjamin La obra de arte en la época de su reproductibilidad técnica ${ }^{2}$, escrito en 1935 y publicado un año después. Sin embargo, algunos de los temas tratados en el ensayo ya los había teorizado antes de una forma u otra; el mismo Adorno era consciente de ello cuando, comentando el texto en una carta escrita a Benjamin, dice que en El origen del "Trauerspiel" alemán ya encontraba ideas que apuntaban hacia algunas reflexiones de La obra de arte (Adorno 1995, p. 139). No en vano, el joven Adorno admiraba El origen del "Trauerspiel" alemán de Benjamin hasta tal punto que le había ofrecido un seminario en su primer año como profesor en la Universidad de Frankfurt ${ }^{3}$ (Wolin 1994, p. 166). Pese a la continuidad que observa Adorno entre esas dos obras, también critica el marxismo excesivamente mecánico de La obra de

A partir de ahora La obra de arte.

Según Susan Buck-Morss (1981), la influencia de Benjamin sobre Adorno supera incluso la de Horkheimer. 
arte y otras obras de la época, como El París del Segundo Imperio en Baudelaire ${ }^{4}$, y trata de hacer ver a Benjamin la necesidad de volver al modelo de análisis del $E l$ origen del "Trauerspiel" alemán. Según él, pese a no utilizar una terminología tan explícitamente marxista era "un marxismo mejor" (Adorno 1995, p. 156). Horkheimer también fue de similar parecer, cuando indujo a Benjamin en el proceso de edición de La obra de arte a cambiar algunos conceptos de cariz marcadamente marxista ${ }^{5}$ y suprimir algunas partes (en especial el prólogo en su totalidad), cambios que Adorno declaró compartir en 1968 (Schmucler 1993, p. 240). Con todo, sería incorrecto definir a Benjamin como un marxista positivista; no en vano, las otras dos figuras con las que Benjamin debatió intensamente en aquella época, a saber, Gershom Scholem y Bertolt Brecht, le reprochaban lo contrario, su misticismo. Es cierto que a partir de los años 30 Benjamin comienza a incorporar nociones y argumentaciones marxistas a su teoría, que tenía ese cariz místico desde sus primeras obras, pero para Scholem y Brecht, aún en los textos que Adorno consideraba excesivamente mecánicos, Benjamin derivaba en exceso hacia lo místico. En palabras de Brecht, "All mysticism, under the guise of anti-misticism. This is the form in which the materialist conception of history is adapted! It is fairly dreadful" (citado en Wolin 1994, p. 141). No en vano, el texto de Benjamin fue rechazado por la revista Das Wort, del que Brecht era coeditor. Adorno, sin embargo, refiriéndose a El París, proponía resolver el contraste entre esos dos polos por medio de la teoría: "el trabajo se encuentra en el cruce entre magia y positivismo. Este pasaje está embrujado. Solo la teoría podría romper el hechizo: su propia teoría, sin consideraciones, bien especulativa" (Adorno 1995, p. 155). De hecho, en la carta de Adorno del 18 de marzo de 1936 donde criticaba La obra de arte por su ortodoxia marxista, dicha ortodoxia la achacaba a la influencia de Brecht ${ }^{6}$, y deseaba que "el sol de Brecht vuelva a sumergirse en aguas exóticas" (Adorno 1995, p. 145).

En el debate, así como en el texto de Benjamin en su conjunto, el concepto de aura es un elemento central. Pese a que el concepto a menudo se ha interpretado como una suerte de característica que tienen las obras de arte (religiosas y modernas), algunos teóricos (Rosen 2004; Bürger 2010) han comentado que, fundamentalmente, es un concepto referido a la recepción de la obra, es decir, a la forma en que experimentamos las obras. En efecto, ello parece confirmarse si tenemos en cuenta que a lo largo del texto hay una consideración continua del polo de la recepción, a través de otros conceptos como la "atención distraída" y la "estetización de la política". No en vano, la propia conceptualización del aura viene precedida de algunas consideraciones sobre la percepción, en las que se subraya la importancia de la historia a la hora de analizar la cuestión (Benjamin 2003, p. 46). Dicho de otra forma, Benjamin aboga por la historización del concepto de percepción. Esta idea, que viene a criticar y completar

A partir de ahora El París.

Los conceptos "comunismo" y "fascismo" fueron reemplazados, respectivamente, por "las fuerzas constructivas de la humanidad" y "el estado totalitario" (Wolin 1994, p. 183)

Buck-Morss (1981) representa las diferencias entre Adorno y Benjamin a través del paulatino acercamiento del primero hacia Horkheimer y del segundo hacia Brecht. 
el idealismo kantiano, aparece ya en sus primeros escritos, y de forma claramente explícita en "Sobre el programa de la filosofía venidera".

Exigencia de la filosofía venidera: crear, sobre la base del sistema kantiano, un concepto de conocimiento al que corresponda un concepto de experiencia de la que el conocimiento sea teoría. Y así, o a esa filosofía se le podría llamar "teología" estudiada en su parte general o la teología quedaría subordinada a ella en la medida en que contiene elementos filosóficos históricos. Experiencia será, en consecuencia, la multiplicidad continua y unitaria de lo que es el conocimiento (Benjamin 2007, p. 172).

En ese sentido, aun el Benjamin más místico tendría una base epistemológica fundamentalmente kantiana (Rosen 2004), en la medida en que quiere ensanchar ésta para incluir algunos tipos de experiencias que la cultura cientifista habría menospreciado. El concepto de aura, pues, se inscribe en esa preocupación de Benjamin en torno a la percepción, la experiencia, y en el intersticio entre el materialismo y el misticismo.

¿Qué es propiamente el aura? Un entretejido muy especial entre espacio y tiempo: aparecimiento único de una lejanía, por más cercana que pueda estar. Reposando en una tarde de verano, seguir la línea montañosa en el horizonte o la extensión de la rama que echa su sombra sobre aquel que reposa, eso quiere decir respirar el aura de esas montañas, de esa rama (Benjamin 2003, p. 47).

En primer lugar, es llamativo observar que el primer ejemplo que ofrece para caracterizar el aura no tiene que ver con el campo del arte. Por ello, se debe subrayar que el aura transciende el objeto artístico y remite a un tipo de percepción más general. No en vano, en la tercera y última versión del manuscrito, encontrado en los años 80 en el archivo de Max Horkheimer, Benjamin distinguía entre el aura de los objetos históricos y el aura de los objetos naturales. De modo que, en una línea netamente kantiana ${ }^{7}$, la percepción estética transciende el objeto artístico, cuestión que le permitirá desarrollar también sus reflexiones sobre la estetización de la política. En el caso de los objetos artísticos modernos, Benjamin identifica en el aura una suerte de prolongación de un elemento de los objetos artísticos antiguos -mágicos y religiosos-, que el proceso de secularización no logró eliminar. El aura, así, sería el garante de la autenticidad y el carácter único de la obra de arte. Sin embargo, con las transformaciones tecnológicas y el advenimiento de la reproductibilidad técnica, el aura entra en decadencia, y con él, la autenticidad y unicidad de los objetos artísticos.

Por primera vez en la historia del mundo la reproductibilidad técnica de la obra de arte libera ésta de su existencia parasitaria dentro del ritual. La obra de arte reproducida se vuelve en medida creciente la reproducción de una obra de arte

Kant abre por primera vez de forma sistemática en la historia de la filosofía del arte, con su giro subjetivo, la posibilidad de que la experiencia estética no esté limitada al objeto artístico, como puede verse con facilidad en sus analíticas de lo bello y lo sublime. 
compuesta en torno a su reproductibilidad. De la placa fotográfica es posible hacer un sinnúmero de impresiones; no tiene sentido preguntar cuál de ellas es la impresión auténtica. Pero si el criterio de autenticidad llega a fallar ante la producción artística, es que la función social del arte en su conjunto se ha trastocado. En lugar de su fundamentación en el ritual, debe aparecer su fundamentación en otra praxis, a saber: su fundamentación en la política (Benjamin 2003, p. 51).

Es así como Benjamin propone la distinción entre el valor de culto, propio de las obras de arte auráticas, y el valor de exhibición, propio de las obras de arte postauráticas. Si para las obras de arte religiosas y mágicas lo fundamental era su existencia como objetos de culto, con la reproductibilidad técnica y la pérdida de la noción de autenticidad, las obras de arte obtienen una capacidad de exhibición nunca antes conocida, perdiendo su valor de culto.

Se puede decir que, con matices, Adorno está de acuerdo con Benjamin en el proceso histórico general esbozado por el segundo (Wolin 1994, p. 193), pero mientras que éste observa la posibilidad de un nuevo arte político en la decadencia del aura, Adorno advierte del peligro de que "el mundo administrado" irrumpa en el campo del arte. De ahí la conocida frase a través de la cual Adorno resumió su discrepancia con Benjamin: "Lo que yo postularía sería un plus de dialéctica" (Adorno 1995, p. 143). Adorno valora como excesivamente reduccionista el esquema benjaminiano de polos contrapuestos entre el arte aurático y postaurático. En el caso del arte aurático, Adorno observa un momento positivo que le permite resistir, desde su autonomía ${ }^{8}$, al mundo administrado. En el caso del arte postaurático, identifica la posibilidad de que en lugar de producir un arte político radical y rupturista, caiga en la cooptación y la afirmación de lo dado. Lo que Adorno viene a reclamar es una caracterización del proceso de pérdida de aura más dialéctica, en el que no se pase ingenuamente de un arte teológico (así llama Benjamin a la doctrina del art pour l'art) a un arte político emancipatorio.

Con todo, ya se pueden observar discrepancias entre los dos en la caracterización del concepto de aura, que se fundamentan en la distinta concepción de la noción de $S_{c h e i n}{ }^{9}$ (Rosen 2004). Para Adorno, el aura no es sino un nuevo término para denominar el Schein (Adorno 1995, p. 139). En la tradición idealista alemana, el Schein es la señal donde se manifiesta la verdad estética, la puerta de acceso al arte. No se trata de una representación, sino de la plasmación (presentación) de "la verdad en forma sensible" como lo caracterizaba Adorno (Wellmer 1993, p. 19). Bajo esta concepción, en gran medida paradójica, la obra de arte da acceso a una verdad transcendente que va, por tanto, más allá de su percepción sensible. Por eso se le llama "símbolo de transcendencia" (Rosen 2004, p. 12), en contraposición a la alegoría, que guardaría una representación

8 Adviértase que en el caso de Adorno, la autonomía del arte siempre es relativa, como se puede apreciar fácilmente en el hecho de que lo distancia del art pour l'art (2004, p. 224). No en vano, su distinción entre la estética y el esteticismo es clave para entender el camino que traza para superar la dicotomía planteada por Benjamin (Kaufman 2005, p. 124). En castellano se suele traducir como "símbolo". 
convencional ${ }^{10}$. Nos encontramos, así, ante una aporía: en el conocimiento no discursivo del arte, la verdad aparece de forma sensible pero en la fugacidad de la experiencia estética, cosa que lo hace imposible de captar. La solución que ofrece Adorno para desenredar esta aporía es la filosofía, y por eso escribió en su Teoría estética la célebre sentencia de que la verdad de la obra de arte solo se puede extraer a través de la filosofía: "El contenido de verdad de las obras de arte es la resolución objetiva del enigma de cada una. Al reclamar una solución, el enigma remite al contenido de verdad. Este solo se puede obtener mediante la reflexión filosófica" (Adorno 2004, p. 221). Esta referencia a la filosofía no es sino la apelación al trabajo hermenéutico necesario para la elucidación del contenido de verdad de las obras.

Pero si la verdad que contiene la obra de arte quedara encerrada en el momento de la experiencia estética, se perdería, y la experiencia estética sería una nadería. De ahí que las obras de arte, a causa de lo que en ellas señala más allá del momento fugaz de la experiencia estética, se vean remitidas a la "razón interpretativa", a que la interpretación exponga la verdad que contienen: para Adorno, interpretación significa interpretación filosófica; la "necesidad de interpretación" que las obras tienen es la necesidad que la experiencia estética tiene de aclaración filosófica (Wellmer 1993, p. 19).

Lo que se descubre a través de ese trabajo hermenéutico es el Geist, y ahí radica la aportación adorniana a la tradición idealista. Para los idealistas, el contenido de verdad de la obra de arte, su Geist, sería original e independiente, manifestación de la unicidad absoluta de la obra; para Adorno, sin embargo, está estrechamente ligada a las fuerzas productivas (Rosen 2004). Ése sería el momento materialista de Adorno, el cual, como se ve, no elimina el Schein, sino que da forma material a su contenido de verdad a través del Geist.

La concepción materialista del arte de Benjamin y Adorno se distancian justamente en ese punto. Benjamin plasma la abolición del aura, del Schein (según la lectura adorniana), como vestigio de una estética idealista que debe ser superada por una lectura materialista; Adorno, sin embargo, trata de rescatar el Schein y su contenido de verdad (que permite resistir al mundo administrado) haciendo una lectura materialista de su interior (del Geist). Benjamin confía en la posibilidad de un arte político prescindiendo del aura, Adorno lo ve improbable por la fuerza de la industria cultural y la lógica capitalista que no deja alternativa entre un arte aurático autónomo que resista al mundo administrado y un arte postaurático político necesariamente panfletario y cooptado. Michel Rosen caracteriza esta divergencia a través de la adscripción de cada pensador a una línea marxista diferente: el marxismo kantiano de Benjamin ${ }^{11}$ frente el marxismo hegeliano de Adorno.

10 Esta es una idea común en el idealismo (Goethe, Schelling, Coleridge) que no comparte Benjamin, el cual trató de dignificar la alegoría en El origen del "Trauerspiel" alemán.

11 La línea kantiana de Benjamin se remonta hasta sus inicios, cuando pretendía elaborar una "teoría cognitiva sobre bases kantianas, que pudiera dar cuenta tanto de la experiencia filosófica como de la experiencia místico-religiosa (Buck-Morss 1981, p. 63). 
De hecho, Adorno desarrollará las ideas críticas con la industria cultural en los años siguientes al debate con Benjamin, especialmente en "Sobre el carácter fetichista de la música y la regresión" publicado en 1938, y en el capítulo "La industria cultural" de la Dialéctica de la Ilustración ${ }^{12}$ publicado por primera vez con el nombre Fragmentos filosóficos en 1944 y con su nombre definitivo en 1947. En ambos textos se ven intensificados los recelos respecto al tema mostrados a Benjamin en la carta de 1936, empujado en parte por lo visto y vivido en los Estados Unidos, a donde se exilió en febrero de 1938 y donde permaneció hasta octubre de 1949. En la primera crítica se centraba en la música y en la creciente mercantilización de esa disciplina artística.

El concepto de fetichismo musical no ha de deducirse psicológicamente. Que se consuman "valores" y que éstos porten consigo afectos, sin que sus cualidades específicas sean alcanzadas por la conciencia del consumidor, es una expresión ulterior de su carácter de mercancía. Pues la totalidad de la vida musical del presente está dominada por la forma de la mercancía: se han erradicado los últimos residuos precapitalistas. La música, con todos los atributos de lo Estético y de lo Sublime que le son otorgados generosamente, está en América esencialmente al servicio de los anuncios de las mercancías que han de adquirirse para poder oír música (Adorno 2009, pp. 24-25).

Para Adorno, la música ligera o comercial, y, en general, la industria cultural, hacen de la obra de arte una potente arma para alienar a las masas. En la Dialéctica de la Ilustración defenderá justamente que la reproductibilidad técnica, lejos de emancipar a los receptores, como veía posible Benjamin, es un aliado del mundo administrado.

La abolición del privilegio cultural por liquidación no introduce a las masas en ámbitos que les estaban vedados; más bien contribuye, en las actuales condiciones sociales, justamente al desmoronamiento de la cultura, al progreso de la bárbara ausencia de toda relación. (...) El arte ha mantenido al burgués dentro de ciertos límites mientras era caro. Pero eso se ha terminado. Su cercanía absoluta, no mediada ya más por el dinero, a aquellos que están expuestos a su acción, lleva a término la alienación y asimila a ambos bajo el signo de una triunfal reificación. (Adorno 1995, p. 205)

Es así como en la era postaurática, Adorno percibe la reificación de la cultura, en donde el valor intrínseco de la cultura queda vaciada por la mercancía (Wolin 1994, p. 196). Solo a través de rescatar el aura y su autonomía puede salvarse el potencial político de la obra de arte como resistente al mundo administrado. La divergencia entre ambos teóricos también tendría relación con las referencias artístico-literarias de ambos. Mientras Adorno observaba un línea crítica en el arte aurático desde el alto romanticismo hasta

12 El libro en su conjunto lo escribieron, como es sabido, Adorno y Horkheimer, pero de la siguiente forma: cada capítulo fue escrito en su totalidad por uno de ellos, mientras que el otro lo revisaba y, a veces, lo reescribía. El primer borrador del capítulo "La industria cultural" fue escrito por Adorno, y se considera una parte netamente adorniana. 
el modernismo, Benjamin estaba interesado en las corrientes más vanguardistas de la época, especialmente en las más anti-institucionales como el dadaísmo. En ese sentido, es especialmente relevante el concepto de shock que desarrolla en La obra de arte, concepto que recoge directamente de los dadaístas. Ello, junto a la divergencia sobre Brecht, ilustra muy bien las diferentes visiones estéticas de uno y otro.

Volviendo a la cuestión del Schein y el Geist, algunos teóricos (Kaufman 2005) han advertido que el mismo Benjamin asumió la ligazón entre aura y las fuerzas productivas teorizada por Adorno, en sus obras posteriores sobre Baudelaire ${ }^{13}$. Héctor Schmucler ha argumentado algo similar añadiendo la Tesis de la filosofía de la historia a esas obras. Según esta tesis, Benjamin se habría percatado en los últimos años de su vida que la técnica, lejos de llevar a esa suerte de politización (emancipatoria) del arte, había contribuido al esteticismo de la política, peligro que, por otra parte, ya se contemplaba en el epílogo de La obra de arte.

Con todo, hay que tener en cuenta que el debate entre Adorno y Benjamin no puede analizarse en la actualidad obviando la cuestión de la recepción de dicho debate. El contexto de dicha recepción es el debate de la posmodernidad a partir de los años 80, y, efectivamente, el debate original, que se dio en los años 30 y 40, adquirió una dimensión diferente medio siglo después. Por un lado, a partir de los movimientos estudiantiles de finales de los 60, respecto a los cuales Adorno, como es sabido, fue reacio, emergió un arduo debate, especialmente en Alemania, acerca del modo en que Horkheimer y Adorno supuestamente transfiguraron la obra de Benjamin para adaptarla a sus tesis. Según esta crítica, aquellos trabajos de edición hicieron parecer la obra de Benjamin menos marxista de lo que realmente era (Moore 2012). Unos años más tarde, una de las investigadoras más relevantes de la teoría crítica, Susan Buck-Morss, argumentó en su libro referencial Origen de la dialéctica negativa, que dicha interpretación era parcial y engañosa. Las ideas de Benjamin, y muy especialmente La obra de arte, cobraron un nuevo sentido en la posmodernidad, a través de aquellos que Beatriz Sarlo llamó "partidarios de Benjamin", es decir, aquellos que interpretaron la contemporaneidad por medio de sus ideas ${ }^{14}$. Entre ellos fueron muy influentes los teóricos de la posmodernidad más afirmativa, la cual entendió la teoría de la reproductibilidad técnica benjaminiana como una suerte de celebración del desarrollo tecnológico y una aceptación acrítica de sus bondades. Es así como se ha presentado un Benjamin integrado con el desarrollo del capitalismo tardío. Frente a él, se situaría precisamente Adorno, como representante de una concepción elitista de la cultura en que defendería a toda costa la autonomía estética y el modernismo estético (Kaufman 2005, p. 122). La paradoja consiste en que la posmodernidad afirmativa rescató justamente el Benjamin más ortodoxamente marxista, y que fue precisamente Adorno el primero que criticó dicha ortodoxia. No son pocos los que han denunciado las distorsiones que ha generado la historia de la

13

14

Sobre algunos motivos en Baudelaire y El París del Segundo Imperio en Baudelaire.

A estos partidarios, Sarlo contrapone los “comentaristas de Benjamin”, los cuales, en lugar de estirar sus ideas hasta la actualidad, tratarían de realizar una lectura más filológica de sus textos, adscritos fundamentalmente al contexto en que fueron escritos. 
recepción de este debate, y se han opuesto a la recepción posmoderna dominante de Benjamin (Costello 2005). Algunos partidarios de Benjamin lo han formulado en términos harto crudos.

Hemos intentado hacerla menos legible [la obra de Benjamin] para las estrategias tranquilizadoras del espíritu posmoderno. (...) El Benjamin complaciente que circula por los pasillos de la cultura masiva, que adorna las teorías extasiadas en la fugacidad de lo efímero, en el imperio de lo mediático, en la exaltación del consumo, no es nuestro. Recordar el marxismo de Benjamin no es sólo un ejercicio de exégesis: pretende una reivindicación de la memoria en tiempos de un olvido indiferenciado (Schmucler 1993, p. 248).

No obstante, no solo ha sido rescatado Benjamin por sus partidarios en la contemporaneidad. Según Robert Kaufman, la vía negativa de Adorno (y en los últimos textos de Benjamin) que reivindica una suerte de arte aurático crítico, también ha tenido sus seguidores, no únicamente a nivel teórico sino a nivel artístico. Tal y como defiende él, en la obra de numerosos artistas contemporáneos de diferentes disciplinas (artes visuales, artes performativas, literatura, música) se puede observar una relectura productiva del modernismo aurático que acabaría con el mantra posmoderno del fin del modernismo (y del aura).

\section{El problema del kitsch}

El debate en torno al kitsch se dio paralelamente al de la reproductibilidad técnica y se desarrolló en parte por los mismos autores, aunque apenas se hayan trazado relaciones entre ambos. En efecto, tanto Adorno como Benjamin aportaron ideas al debate del kitsch. Benjamin trató el tema en diferentes textos a lo largo de toda su vida ${ }^{15}$, siendo su primera publicación sobre el tema el temprano ${ }^{16}$ "El kitsch onírico, glosa sobre el surrealismo", publicado en 1927 (aunque escrito en 1925) y perteneciente, por tanto, a los inicios de tendencia más mística de Benjamin. Nótese que hay un hilo conductor ya entre algunas características de ambos debates en Benjamin. La cuestión del kitsch viene íntimamente relacionada con el surrealismo, mientras que sus ideas sobre la reproductibilidad técnica lo están con los dadaístas y su teoría del shock. Por su parte, Adorno trató el kitsch en más textos y más transversalmente, a menudo, muy estrechamente ligado a sus teorías sobre la música, cosa que también sucede, como ya se ha visto, con la cuestión de la reproductibilidad técnica en "Sobre el carácter fetichista de la música y la regresión".

15 Incluso caracterizó su Libro de los Pasajes como un trabajo sobre el kitsch (Menninghaus 2009, p. 39)

16 El primer libro dedicado al kitsch data de 1925, el Der Kitsch. Eine Studie über die Entartung der Kunst de Fritz Karpfen. 
El debate se despliega, en un primer término, en los años 20 y 30 del siglo XX. Además de los citados anteriormente, cabe destacar los textos de Hermann Broch y Norbert Elias. Pese a que el debate se produjera fundamentalmente en el contexto germano, su repercusión traspasó sus fronteras, como lo muestra el ya mencionado "Vanguardia y kitsch" de Clement Greenberg. Después de que a lo largo de unas décadas el tema dejara de estar en el primer plano de los debates estéticos, a partir de los años 60 el advenimiento de la posmodernidad lo revitalizó, y permitió realizar nuevas lecturas, como se verá más adelante.

El kitsch comparte con la cuestión de la reproductibilidad técnica la centralidad que le da al polo de la recepción. Es decir, el kitsch, pese a tener una evidente objetividad (Adorno 2011, p. 824), se define fundamentalmente por medio de la recepción. En palabras de Abraham Moles, "[el kitsch] es uno de esos tipos de relación que mantiene el hombre con las cosas, un modo de ser más que un objeto o aún un estilo" (1971, p. 11). No en vano, el intento más analítico de definir el kitsch, realizado por Thomas Kulka, nos muestra también esa importancia. De las tres condiciones que establece para definir el kitsch, dos de ellas, la segunda y la tercera, pertenecen claramente al campo de la recepción, y en el primero hay una alusión implícita.

1. El kitsch representa un objeto o tema que se tiene comúnmente por bello o emotivo.

2. El objeto representado es rápida y fácilmente reconocible.

3. El kitsch no enriquece sustancialmente nuestras asociaciones mentales ligadas al objeto representado (2011, p. 25).

Es por ello que, a menudo, el kitsch se relaciona con la cuestión del gusto ${ }^{17}$, en la medida en que representaría justamente el mal gusto de la clase media frente al exquisito gusto de la élite (Elias 2011, p. 41). Pese a que el kitsch se ha relacionado con el gusto de las clases populares, hay que señalar que el fenómeno es fundamentalmente burgués y de clase media, pese a que con el tiempo se haya convertido "en el estilo de vida ideal de toda la sociedad" (Calinescu 2003, p. 240). Greenberg defendió lo propio cuando indicó que el kitsch, definido como "cultura sucedánea", estaba destinada, según él, "a aquellos que, insensibles a los valores de la cultura genuina, estaban hambrientos de distracciones que sólo algún tipo de cultura puede proporcionar" (Greenberg 2002, p. 18).

Adorno también siguió esa línea cuando definió el kitsch, reelaborando el concepto aristotélico par excellence referido a la recepción, como "parodia de catarsis" (Adorno 2004 , p. 316). Como es común en todos los análisis adornianos, relaciona tanto la crítica inmanente de la obra como la de la recepción con una crítica sociológica, lo cual ofrece

$17 \quad$ En una de las antologías referenciales sobre el tema, se ve ya en el título esa identificación entre el kitsch y el mal gusto: Kitsch: An Anthology of Bad Taste de Gillo Dorfles. 
una perspectiva más general y completa de la problemática ${ }^{18}$. Es así como la crítica del kitsch tiene en Adorno al más feroz de los críticos, aunque ello no quiera decir que simplifique la cuestión. De hecho, en uno de los textos sobre el tema, Adorno achaca a Thorstein Veblen no haber considerado "la necesidad histórica" (Adorno 2008, p. 73) del kitsch, que está, para Adorno, estrechamente ligado a la modernidad. Veblen entiende el kitsch como mero anacronismo ${ }^{19}$, mientras que Adorno ve en él una manifestación genuinamente postindustrial, y por ello, pese a que lo critique radicalmente, no lo desdeña en absoluto, en la medida en que también ofrece claves para entender el arte genuino. Más aún, Adorno llega a plantear, en su empeño dialéctico, que el concepto de arte tiene su momento kitsch, que indica el aspecto social de la obra de arte (Adorno 2004, p. 163).

Es en ese continuo afán de Adorno por ligar la estética con la crítica sociológica donde plantea, en una clara línea marxista, que la función social del kitsch es la de "engañarles [a las gentes] sobre su verdadera situación. Transfigurar su existencia, hacer que metas que son gratas a cualesquiera poderes se les aparezcan con un resplandor de cuento de hadas" (Adorno 2011, p. 826). La distracción mencionada por Greenberg tendría justamente esa función ideológica ${ }^{20}$. Por ello, es claro que el kitsch es para Adorno fundamentalmente ideológico, y que funciona, por tanto, como distracción de las clases subalternas que frena su fuerza revolucionaria. Con todo, la insistencia adorniana en la dialéctica permite trazar la idea de que un objeto estético no puede ser kitsch en sí, es decir, que la valoración como tal está sujeta al devenir histórico. El kitsch solo emerge, por tanto, a través de la crítica histórica, de tal modo que "no existe ningún criterio universal" (Adorno 2011, p. 828). Más aún, plantea la posibilidad de que "el mismo discurso sobre el kitsch comienza a convertirse en kitsch al sucumbir a la dialéctica histórica de la que emergió su objeto" (Adorno 2011, p. 828). Esta dimensión histórica es fundamental para comprender el kitsch, ya que su existencia no se justifica por medio de ciertas características formales de los objetos estéticos, sino a través del devenir histórico y las nuevas formas de recepción. Una de esas frases aforísticas típicas adornianas da buena cuenta de ello: "Lo que fue arte puede llegar a ser kitsch" (Adorno 2004, p. 417). Y de ahí que en otro momento de la Teoría estética lo caracterice como aquello que fue bello "en otros tiempos" (Adorno 2004, p. 70). Así, el kitsch, siempre aparece en estilos o formas artísticas convertidas

18 Greenberg también observa en la misma época la necesidad de analizar el kitsch en todas sus vertientes: "La respuesta [sobre el carácter del kitsch] exige algo más que una investigación estética. En mi opinión, es necesario examinar más atentamente y con más originalidad que hasta ahora la relación entre la experiencia estética -tal y como se enfrenta a ella el individuo concreto, y no el generalizado-y los contextos históricos y sociales en los que esa experiencia tiene lugar" (Greenberg 1979, p. 12).

19 Veblen seguiría aquí la concepción primigenia del kitsch, propuesta por Frank Wedekind, como "barroco o gótico de nuestro tiempo" (Adorno 2008, p. 73).

20 Para una profundización en el cariz marxista de las ideas estéticas de Greenberg, y, sobre todo, su conexión con Adorno, ver "Modernism and Marxism, Greenberg and Adorno" de Ian McLean. 
en lugares comunes o convencionales, en formas que no producen ninguna dificultad o perturbación de recepción en la época contemporánea. Esta es una idea común en la mayoría de los críticos del kitsch, y también en Broch.

El kitsch siempre está sometido a la influencia dogmática de lo que "ya ha existido", para comprender que nunca toma directamente sus vocablos de la realidad del mundo, sino que utiliza vocablos prefabricados que con su poder se hacen rígidos hasta convertirse en clichés (Broch 1970, p. 10).

Esta tendencia amable de sujetarse a aquello ya conocido lo relaciona Broch con la función del kitsch de aplacar la nostalgia, que a su vez supone una huida del mundo real (Broch 1970, p. 11). El kitsch propone un mundo estereotipado y de valores fijos y establecidos que ofrece alivio, frente al caos y el conflicto del mundo real.

Así, para Adorno, y siguiendo esta tradición crítica, el kitsch pertenecería al campo de la cultura y no del arte, en tanto que en la teoría adorniana, la cultura la conformarían justamente aquellas obras institucionalizadas o integradas por el mundo administrado; la cultura es para él la esfera de lo establecido, del lugar común. Siguiendo el análisis dialéctico mencionado anteriormente, Adorno plantea una radical historicidad de las obras, las cuales pueden convertirse en cultura toda vez que hayan sido domesticadas. Por ello, plantea que cualquier planteamiento artístico que defienda "categorías inalienables" tiene el riesgo de caer en lo kitsch (Adorno 2004, p. 202), justamente porque obvia la historicidad de la obra de $\operatorname{arte}^{21}$. El esfuerzo de la teoría adorniana consiste en tratar de reivindicar nuevas formas artísticas que pongan en jaque y renueven la cultura integrada; una reivindicación complicada en unos tiempos en los que nada relacionado con el arte parece obvio (Adorno 2004, p. 9). Este aspecto histórico del kitsch como repetición de formas estéticas trilladas también lo contempla Kulka.

La necesidad de la identificación inmediata y sencilla impide, por el contrario, la innovación estilística. El kitsch nunca se asoma a las vanguardias. El kitsch nunca se atreve con estilos que aún no han sido universalmente digeridos. Podrá sumarse al carro solo cuando la novedad se convierta en banalidad. Esto explica, a mi entender, el carácter ultra-conservador, y estilísticamente reaccionario, del kitsch (Kulka 2011, p. 19).

La contraposición de las vanguardias con el kitsch es algo en lo que incidió Greenberg en su influyente artículo de 1939, donde identificó el kitsch con la retaguardia (Greenberg 2002, p. 17). La distinción fundamental la estableció a partir del concepto clásico de mimesis de Aristóteles: "si la vanguardia imita los procesos del arte, el kitsch imita sus

21 Adorno plantea el problema en el contexto de una crítica del idealismo y su concepción de eternidad en el arte; tanto es así que define como kitsch el epitome del idealismo, el arte por el arte (Adorno 2004, p. 313). Además, añade que ése es el problema de cualquier purismo, y que Brecht también tuvo el mismo problema ligado al arte social. No es casual que Brecht aparezca en ambos debates como objeto de las críticas de Adorno. 
efectos" (Greenberg 2002, p. 22). Es una formulación que incide en la importancia del polo de la recepción para entender el kitsch, además de que saca a relucir la concepción formalista y objetualista radical de Greenberg, donde las cuestiones como "los efectos" se vuelven irrelevantes, o incluso perjudiciales, para el análisis crítico del arte.

En ese punto es donde mejor se observa la conexión entre la cuestión de la reproductibilidad técnica y el kitsch. La reproductibilidad técnica ahonda, con su pérdida del aura, en la banalización de la recepción, y así, se acelera la integración de las obras de arte. La reproductibilidad técnica permitiría, siguiendo el argumento de Adorno, una mayor y más rápida neutralización del potencial transformador del arte, en la medida en que facilitaría la culturización, en términos adornianos, de la esfera del arte. El kitsch, por medio de la reproducción técnica, extraería a las obras de arte su dimensión estética.

Una vez que el kitsch es técnicamente posible y económicamente rentable, la proliferación de imitaciones baratas o no tan baratas de todo - desde arte primitivo o popular hasta la última vanguardia-está únicamente limitada por el mercado. El valor se mide directamente por la demanda de réplicas o reproducciones espúreas de objetos cuyo original significado estético consistía, o debería haber consistido, en ser únicas y, por tanto, inimitables (Calinescu 2003, p. 224).

Así, siguiendo una línea adorniana, se podría decir que el cambio del valor de culto al valor de exhibición que Benjamin teoriza en el contexto del debate sobre la reproductibilidad técnica se materializa en una transformación del valor de uso al valor de cambio, ejemplificado en el kitsch. Si las obras de arte auténticas se definen por su valor de culto -huella del arte religioso-y su valor de uso-forma de recepción única-, el kitsch lo hace por su valor de exhibición -con el aumento de las réplicas-y su valor de cambio - con el aumento del mercado-.

De este modo, tal y como sucede con la cuestión de la reproductibilidad técnica, el problema del kitsch está íntimamente unido al tema de la autonomía estética, en la medida en que sería un representante paradigmático del arte heterónomo. Asumiendo que el kitsch es una forma artística típica de la industria cultural, es evidente que en la teoría estética de Adorno aparece como antítesis del arte autónomo. También está muy presente esta idea en otro texto de la época ya mencionado, concretamente de 1933, de Broch. En él, se traza la diferencia entre el arte autónomo y el arte que "se deja dominar por una influencia externa", es decir, el kitsch (Broch 1970, p. 7). El kitsch es aquel arte subordinado a otras tendencias extra-artísticas, concretamente, subordinado al efecto. Broch es, así, el teórico que con más vehemencia criticó el kitsch como forma artística del efecto y definió su proceder como "substitución de la categoría ética con la categoría estética", en la que "impone al artista la obligación de realizar, no un "buen trabajo" sino un trabajo "agradable: lo que más importa es el efecto" (Broch 1970, p. 9).

Frente a esta línea de crítica radical del kitsch, en la que Adorno es una de las referencias más importantes, Benjamin ofreció una perspectiva diferente. Tal y como sucedería con el debate de la reproductibilidad técnica, Benjamin identificó elementos productivos en el kitsch, y no lo rechazó de plano como arte reaccionario. Para entender 
su posición hay que volver otra vez sobre un concepto clave en Benjamin, que también lo es para comprender la cuestión de la reproductibilidad técnica, a saber, el concepto de experiencia. Es bien sabido que dicha noción, en especial en lo que se refiere a su gradual empobrecimiento histórico (Benjamin 1973) y su repercusión en el arte (Benjamin 2009a), es una de las preocupaciones fundamentales de toda la obra benjaminiana, y fundamental también, como ya se ha visto, para entender su noción de aura. La epistemología de Benjamin, pese a no estar inscrita dentro de un sistema filosófico cerrado -cuestión de la que Benjamin (y Adorno) siempre rehuía-, se fundamenta en una crítica de la noción reduccionista de experiencia, a través del concepto Erfahrung.

What Benjamin calls Erfahrung (experience), as distinct from Erlebnisse (conscious experiences), has little to do with the modern scientific concept of empiricism. Experience in Benjamin's sense includes unconscious desires, manual skills, and religious rituals (Menninghaus 2009, p. 44).

En el caso del kitsch, en particular en su primer texto temprano, se observa una voluntad de superar ese concepto cientifista de experiencia por medio del aparato teórico del psicoanálisis. Para Benjamin, el kitsch está conectado a la experiencia del sueño; tanto en el kitsch como en el sueño, las cosas aparecen con su "lado desgastado" (Benjamin 2009b, p. 229), es decir, estereotipados. Pero ese desgaste es dado por la acumulación de las experiencias en la edad adulta, que imposibilitan una forma de experiencia más directa y que en el sueño y en el kitsch se suspenden. Es decir, en el sueño y en el kitsch esas cosas se experimentan en su inocencia, sin el desgaste del hábito. Por eso, Benjamin relaciona el kitsch con una suerte de experiencia infantil, que sitúa a los niños en una posición de "experiential advantage as a result of their incomplete submission to the taboos and laws of the symbolic order" (Menninghaus 2009, p. 41). En palabras del propio Benjamin, "cuando éramos pequeños, aún no existía la protesta angustiosa contra el mundo propio de nuestros padres. Nos mostrábamos superiores en medio de él, como niños. Con lo banal, cuando lo captamos, captamos lo bueno, que se hallaba muy cerca" (Benjamin 2009b, p. 230). Ahí lo banal cumple una función, que sería la de alcanzar las cosas ya desaparecidas; tanto en el sueño (como indagaron los surrealistas) como en el kitsch, lo banal ${ }^{22}$ ofrece el acceso directo a las cosas que el arte no puede ofrecer, porque éste "comienza a dos metros del cuerpo" (Benjamin 2009b, p. 231). Mientras que el gran arte establece una distancia con las cosas, lo banal se introduce en ellas sin barrera alguna, con la inocencia de un niño. Obviamente, esta distancia del arte no es sino la distancia de aquello de lo que teorizará unos años más tarde, la distancia del aura. En ese sentido, el kitsch tendría un elemento afirmativo esencial, en contraste con los planteamientos estéticos de Adorno.

22 En un momento, Benjamin define el kitsch como "última máscara de lo banal” (Benjamin 2009b, p. 231). 
Kitsch offers instantaneous emotional gratification without intellectual effort, without requirement of distance, without sublimation. It usually presents no difficulties in interpretation and has absolutely nothing to do with an aesthetics of negativity. It is unadulterated beauty, a simple invitation to wallow sentiment -in short, a true antidote to any Adorno-type aesthetics of negativity (Menninghaus 2009, p. 41).

No en vano, Benjamin evoca otro de los conceptos clave de su teoría de la reproductibilidad técnica a la hora de explicar la forma de recepción del kitsch. Según él, esa falta de distancia del kitsch hace que su forma de recepción sea la de la atención distraída, tal y como sucede con el cine y la arquitectura. Es por ello por lo que muestra tanto interés Benjamin en su Libro de los pasajes en lo que Menninghaus llama para-arquitectura, es decir, en los elementos ornamentales y los para-objetos envejecidos como kitsch. De ahí también su interés por los pasajes, interiores, o incluso elementos tan controvertidos en la época como la Torre Eiffel, ejemplos todos ellos de recepción distraída, en la medida en que no exigen el recogimiento necesario para observar las obras de arte auráticas. En lo que se refiere a las referencias literarias, conviene tener presente también que muchos de los referentes literarios de Benjamin pueden incluirse dentro de una historia del protokitsch. Tanto simbolistas como Baudelaire y Rimbaud como los surrealistas atacaron el buen gusto burgués de la época y promocionaron, especialmente Baudelaire, una poética de la ruina y el desecho. De ahí que Benjamin defina la modernidad de Baudelaire como "la disolución del aura a través de la "experiencia" del shock" (Benjamin 1998, p. 76), y de ahí también que sus textos sobre el poeta ahondaran en las diferencias que tuvo con Adorno.

De modo que Benjamin sigue a muchos teóricos (Bergson, Dilthey, Klages, Jung) en la idea del paulatino empobrecimiento de la experiencia en el capitalismo tardío, pero a diferencia de la salida conservadora que proponen aquellos, él observa otra vía de salida positiva, a saber, la moda y su carácter efímero. Benjamin no resuelve la pérdida de la tradición y la subsiguiente crisis de la experiencia a través de una suerte de recuperación de dicha tradición; en contraste, asume la moda y su forma efímera como la forma de experiencia típicamente moderna: "Bygone fashions of all things, can act as a substitute for the extinct "forces of tradition" and remembrance" (Menninghaus 2009, p. 47). Lo kitsch, en tanto que placer de lo demodé, también se sitúa así dentro de esta problemática de la experiencia y de la vía productiva que le da a ella Benjamin. No se trataría tanto de aceptar acríticamente el kitsch como de buscar su momento positivo para superarlo.

Además de estos ecos internos entre los dos debates, la recepción de ambos en la segunda mitad del siglo XX es asimismo relevante a la hora de medir las resonancias, especialmente en la posmodernidad. En efecto, no es casual que tanto la cuestión de la reproductibilidad a través de la revalorización de la figura de Benjamin como el problema del kitsch a través de lo camp, renaciesen paralelamente a partir de los años 70. En el contexto estadounidense, la recuperación del pensamiento de Benjamin se dio a finales de esa década, por medio de los pensadores del entorno de la revista October, los que releyeron las ideas de Benjamin para teorizar sobre el arte posmoderno (Costello 2005). Para ello, se acogieron fundamentalmente a sus reflexiones sobre la 
fotografía y la reproductibilidad técnica. Más allá del ámbito estrictamente artístico, Benjamin es frecuentemente citado por los autores que trabajaron la cuestión de la modernidad y la posmodernidad (Jameson 1969; Huyssen 2002). Paralelamente, la cuestión del kitsch también volvió a emerger en el debate cultural internacional. Hasta los años 70, las investigaciones sobre el kitsch se desarrollaron básicamente en el ámbito germano -con la prácticamente única excepción de Greenberg-(Menninghaus 2009), pero a partir de entonces, el kitsch se sitúa en el centro de numerosos debates estéticos contemporáneos. En el contexto posmoderno, se trató de revalorizar tanto la reproductibilidad técnica como el kitsch, que habían sido leídos y criticados por la mayor parte de la teoría crítica como fenómenos de alienación. La recuperación de Benjamin les servía a los nuevos teóricos de la posmodernidad para contrarrestar dicha crítica, en la medida en que Benjamin ya había observado en los años 30 ciertos aspectos productivos de ambos. Con todo, como ya se ha apuntado, esta relectura dominante (Costello 2005, p. 10) ha presentado un Benjamin integrado, despojado de su aspecto ético y político, y de ahí que muchos teóricos hayan denunciado esa tergiversación del crítico alemán. Al fin y al cabo, no debe olvidarse que Benjamin observó aspectos productivos de la reproductibilidad técnica y del kitsch pero siempre desde un punto de vista revolucionario, "politizando el arte" (Benjamin 2003, 99) para la consecución de una sociedad igualitaria y desclasada.

\section{Conclusiones}

La cuestión de la reproductibilidad técnica y el debate sobre el kitsch apuntan la necesidad de analizar los debates estéticos del siglo XX desde una perspectiva integral y sistémica. No en vano, teniendo en cuenta el carácter de la obra de dos de los autores más fundamentales de ambos debates -Adorno y Benjamin-, que son obras complejas e integrales, donde las diferentes problemáticas estético-sociales se analizan de forma interrelacional, no es de extrañar que estos debates puedan pensarse también en íntima correlación.

En este caso, el debate sobre el kitsch puede leerse, en Adorno y Benjamin, como un preludio a la discusión que tendrían a partir de la cuestión de la reproductibilidad técnica. En ambos se observa la importancia del concepto de experiencia. Para Benjamin, tanto la pérdida del aura como la proliferación del kitsch son manifestaciones de un proceso de empobrecimiento de la experiencia, del que trata de sacar a toda costa en los dos casos algún momento revolucionario. Por otro lado, en ambos debates la historicidad resulta fundamental, en la medida en que tanto la pérdida del aura como el advenimiento del kitsch solo pueden entenderse en la evolución histórica de la sociedad capitalista. El cambio de una era aurática a una era postaurática y la caracterización del kitsch como placer de lo demodé da buena cuenta de ello. Además, en los dos debates el polo de la recepción tiene una función fundamental; por un lado, la pérdida del aura no es tanto una característica que dejan de tener las obras, como la pérdida de un modo de recepción de ellas; por otro lado, el kitsch está estrechamente unido al efecto, hasta el punto de que Greenberg lo definió como "imitación del efecto del arte". Por último, en los dos debates, la cuestión de la tecnología es, asimismo, central. La 
reproductibilidad técnica alude, efectivamente, a un avance tecnológico concreto y a sus efectos estéticos y sociales, mientras que el kitsch es posible solo en el momento en que ciertos objetos pueden ser masivamente reproducidos. De ahí que se pueda decir que el kitsch es una de las formas estéticas mayoritarias y más exitosas que trae consigo la reproductibilidad técnica.

Todo ello apunta también en una dirección, a saber, en la necesidad de investigar las conexiones entre diferentes debates estéticos que hasta ahora se han analizado esencialmente de forma aislada. Algunos de los conceptos y problemas teóricos subyacentes en muchos de esos debates son comunes, y profundizar en ellos puede ayudar a dilucidar muchas de las discusiones estéticas concretas, como es el caso de la reproductibilidad técnica y el kitsch.

\section{Referencias bibliográficas}

Adorno, Theodor W. (1995), Sobre Walter Benjamin. Madrid: Cátedra. (2004), Teoría estética. Madrid: Akal. (2008). Crítica de la cultura y sociedad I. Obra completa 10/2. Madrid: Akal.

(2009), Disonancias. Introducción a la sociología de la música. Obra completa 14. Madrid: Akal.

(2011), Escritos musicales V. Obra completa 18. Madrid: Akal.

Benjamin, Walter (1973), "Experiencia y pobreza”, en Walter Benjamin, Discursos interrumpidos I. Madrid: Taurus.

(1998), Para una crítica de la ciencia y otros ensayos. Iluminaciones IV. Madrid: Taurus.

(2003), La obra de arte en la época de su reproductibilidad técnica. México: Itaca.

(2005), Libro de los pasajes. Madrid: Akal.

(2007), "Sobre el programa de la filosofía venidera", en Walter Benjamin Obras. Libro II, vol. 1. Madrid: Abada.

(2009a), "El narrador", en Walter Benjamin, Obras. Libro II, vol. 2. Madrid: Abada.

(2009b), "El kitsch onírico" en Walter Benjamin, Obras. Libro II, vol. 2. Madrid: Abada.

Broch, Hermann (1970), Kitsch, vanguardia y el arte por el arte. Barcelona: Tusquets. Buck-Moss, Susan (1981), Origen de la dialéctica negativa. Madrid: Siglo XXI.

Bürger, Peter (2010), Teoría de la Vanguardia. Buenos Aires: Las Cuarenta.

Calinescu, Matei (2003), Cinco caras de la modernidad. Modernismo, vanguardia, decadencia, kitsch, posmodernismo. Madrid: Tecnos.

Costello, Diarmuid (2005), “Aura, Face, Photography: Re-reading Benjamin Today”, Andrew Benjamin, ed., Walter Benjamin and Art. New York: Continuum. 
Elias, Norbert (2011), "El estilo Kitsch y su época”, en Tomas Kulka (et al.), El kitsch. Madrid: Casimiro.

Greenberg, Clement (1979), "Vanguardia y kitsch", en Clement Greenberg, Arte y cultura. Barcelona: Editorial Gustavo Gili. (2002), Arte y cultura (Ensayos críticos). Barcelona: Paidós.

Horkheimer, Max y Theodor W. Adorno (1994), Dialéctica de la Ilustración. Madrid: Trotta.

Huyssen, Andreaas (2002), Después de la gran división : modernismo, cultura de masas, posmodernismo. Buenos Aires: Adriana Hidalgo.

Jameson, Fredric (1969), Walter Benjamin, or Nostalgia, Salmagundi n 10/11, p. 26.

Kaufman, Andrew (2005), “Aura, Still”, en Benjamin, Andrew (ed.), Walter Benjamin and Art. New York: Continuum.

Kulka, Tomas (2011), "El kitsch”, en Tomas Kulka (et al.), El kitsch. Madrid: Casimiro.

McLean, Ian (1988), "Modernism and Marxism, Greenberg and Adorno", Australian Journal of Art, vol. 7, pp. 97-111.

Menninghaus, Winfried (2009), "On the Vital Significance of Kitsch: Walter Benjamin's politics of Bad Taste”, en Andrew Benjamin y Charles Rice, Walter Benjamin and the Architecture of Modernity. Melbourne: Re.Press.

Moles, Abraham (1971), El kitsch. El arte de la felicidad. Barcelona: Paidós.

Moore, Ryan (2012) "Digital Reproducibility and the Culture Industry: Popular Music and the Adorno-Benjamin Debate", Fast Capitalism, 9.1.

Rosen, Michael (2004), "Benjamin, Adorno and the Decline of Aura" en Fred Rush, ed., The Cambridge Companion to Critical Theory. Cambridge: Cambridge University Press.

Sarlo, Beatriz (2000), "Lectores; comentaristas y partidarios", Siete ensayos sobre Walter Benjamin. Buenos Aires: Fondo de Cultura Económica.

Schmucler, Héctor (1993), "La pérdida del aura: una nueva pobreza humana", en Nicolás Casullo, ed., Sobre Walter Benjamin. Vanguardias, historia, estética y literatura. Una visión latinoamericana. Buenos Aires: Alianza.

Wellmer, Albrecht (1993), Sobre la dialéctica de la modernidad y la posmodernidad. La crítica de la razón después de Adorno. Madrid: Visor.

Wolin, Richard (1994), Walter Benjamin. An Aesthetic of Redemption. Los Angeles: University of California Press. 